\title{
INTELLIGENT INDUSTRIAL INSTRUMENTATION BASED ON SILICON MEMS PRESSURE SENSORS
}

\author{
Miloš Frantlović ${ }^{1}$ \\ Ivana Jokić ${ }^{1}$ \\ Marina Marjanović-Jakovljević2 \\ ${ }^{1}$ University of Belgrade \\ ICTM-CMT, Institute of Chemistry, \\ Technology and Metallurgy, \\ Belgrade, Serbia \\ ${ }^{2}$ Singidunum University, \\ 32 Danijelova Street, Belgrade, Serbia
}

\begin{abstract}
:
Research and development in the fields of MEMS sensors and intelligent industrial instrumentation, performed at the Center of Microelectronic Technologies (ICTM-CMT), have resulted in scientific and technological advances beyond the state-of-the-art. In this paper, we give an introduction to MEMS sensors, as well as to the concept of intelligent industrial instrumentation. The SP-12 silicon piezoresistive MEMS pressure sensor, the newest type developed and manufactured at ICTM, is described in detail. Subsequently, a new method is presented that enables simultaneous highperformance pressure and temperature measurement to be performed using a single piezoresistive MEMS pressure sensor. It is devised using the sensor's mathematical model, based on sensor characterization data. A newly developed intelligent industrial transmitter was used as a hardware platform for experimental validation of the measurement method. The obtained results indicate that the measurement performance level of the existing industrial equipment can be met or exceeded using the described approach. Implications regarding the practical use of the presented method are discussed. Finally, two intelligent industrial instruments based on the SP-12 sensor and the described method are presented: a pressure transmitter and a liquid level transmitter. The performance and reliability of both products have been proven in harsh industrial environments.
\end{abstract}

Key words:

sensor, intelligent instrument, MEMS, pressure, temperature.

Acknowledgment:

This work has been funded by the Serbian Ministry of Education, Science and Technological Development, within the project TR 32008.

\section{INTRODUCTION}

The emergence of micro-electro-mechanical technologies and systems (MEMS), which were mentioned for the first time in 1986 in the USA, started a new era in sensor development, which is likely to last throughout the next decades. New sensor structures based on these technologies can achieve measurement performance comparable or superior to those of conventional sensors, while they are of miniature dimensions and suitable for economical mass-production. Additionally, there are features, effects and mechanisms inherent to micro and nano-structures, which are of interest for sensor realization, while their influence on macroscopic objects is often negligible. One of such effects is adsorption of particles, which is utilized
Correspondence:

Miloš Frantlović

e-mail:

frant@nanosys.ihtm.bg.ac.rs 
as the principle of operation of many contemporary chemical and biological sensors (Frantlović et al., 2013). Mechanical MEMS sensors (e.g. pressure and flow sensors, accelerometers, gyroscopes) are widely used today and have a significant market share. The most common mechanisms for conversion of mechanical deformation into electrical signal are based on piezoresistivity and capacitance change (Balavalad and Sheeparamatti, 2015). Historicaly first and also the most successful MEMS sensors are silicon piezoresistive MEMS pressure sensors. They dominate in automotive industry (the largest market share of pressure sensors today), and are very common in process industry, aerospace and military industry, medical equipment and consumer products. As a result of continuous research and development of silicon piezoresistive MEMS pressure sensors during the last 3 decades at the Center of Microelectronic Technologies of the Institute of Chemistry, Technology and Metallurgy, University of Belgrade, a variety of products, including sensors and industrial instruments based on them, has been realized and brought to market (Djurić et al., 1986; Matović et al., 1991; Djurić, 1995; Frantlović et al., 2007; Smiljanić et al., 2007; Frantlović et al., 2009; Smiljanić et al., 2012; Frantlović et al., 2014).

For telemetry of physical quantities (pressure, temperature, liquid level, flow etc.) in industrial processes, a special kind of electronic measurement instruments is used, usually called industrial transmitters. They are intended for operation in harsh industrial environments and they use robust industrial interfaces for connection with the centralized measurement or control system. In their simplest form, industrial transmitters are electronic devices with analog signal processing and analog output signal (most commonly $4 \mathrm{~mA}$ to $20 \mathrm{~mA}$ ). However, in order to satisfy the ever increasing requirements regarding measurement performance, ease of use, and connectivity, new generations of transmitters emerged, offering digital signal processing, interactive user interface and digital two-way communication. The current generation of industrial transmitters, often called intelligent transmiters, have advanced computing and communication capabilities, which enable them not only to achieve high measurement performance, but also to process data obtained by measuring dfferent quantities, and to perform various additional functions, including self-diagnostics.

A majority of modern pressure transmitters is based on silicon MEMS pressure sensors (piezoresistive or capacitive). The measured pressure is typically within the range from $100 \mathrm{~Pa}$ to $10^{8} \mathrm{~Pa}$. Regarding the measurement performance, an inherent property of all semiconductor- based piezoresistive pressure sensing elements is a significant parasitic temperature sensitivity of their output signal, which must be minimized by using suitable correction techniques. Such techniques evolved from simple methods using resistor networks to complex digital signal processing algorithms (Frantlović et al., 2009; Rivera et al., 2007). Contemporary high-performance intelligent industrial pressure transmitters based on MEMS piezoresistive sensors exhibit relative measurement uncertainty as low as $0.05 \%$ of full scale at reference conditions, and typically $>0.15 \%$ in the full temperature range.

Industrial temperature transmitters typically use temperature probes based on platinum resistance sensing elements or thermocouples, and the measured temperatures are typically within the range from $-270^{\circ} \mathrm{C}$ to $1500^{\circ} \mathrm{C}$. Measurement uncertainty achievable using platinum probes can be much better than $0.5^{\circ} \mathrm{C}$ (IEC 60751,2008$)$, while the same value can be achieved only with the best thermocouples (IEC 60584-2, 1989).

In a typical electric powerplant block there is more than 100 temperature and 50 pressure measurement points. While in other kinds of industrial plants the numbers can be significantly different, measurement of both quantities at the same measurement points is needed in many cases. Although industrial transmitters capable of measuring both quantities exist, they are based on separate sensors or sensing elements for each measured quantity. The existing literature boasts only few examples of simultaneous pressure and temperature measurement performed using a single sensing element (Guo et al., 2006; Doelle, 2011). The authors are unaware of such examples based on silicon piezoresistive MEMS sensing elements.

In this paper, a description is given of an SP-12 silicon piezoresistive MEMS pressure sensor developed and manufactured at the Center of Microelectronic Technologies. Subsequently, a method is described that enables simultaneous high-performance pressure and temperature measurement to be performed using a single SP-12 sensor. The obtained results are then discussed, as well as their implications regarding the practical use of the presented method.

\section{METHOD}

\section{Sensor Description}

The newest of the silicon MEMS piezoresistive pressure sensing elements developed and produced at the Center of Microelectronic Technologies is the ICTM 
SP-12, intended for measurement of absolute or relative pressure from $10^{5} \mathrm{~Pa}$ to $10^{7} \mathrm{~Pa}$ ( 1 bar to $100 \mathrm{bar}$ ) (Matić et al., 2013). The material for its fabrication is a double-sided polished single-crystal n-type silicon wafer with a specific resistivity from $3 \Omega \mathrm{cm}$ to $5 \Omega \mathrm{cm}$. Four p-type piezoresistors are formed by boron diffusion on the surface of the sensor's diaphragm, constituting a Wheatstone bridge. Two piezoresistors are in the radial direction and the remaining two in the transversal direction near the edge of the diaphragm. The diaphragm is square, $1.08 \mathrm{~mm} \times 1.08 \mathrm{~mm}$ in size, fabricated by silicon bulk micromachining. The diaphragm thickness is from $32 \mu \mathrm{m}$ to $160 \mu \mathrm{m}$, depending on the nominal pressure range of the sensing element. The position of the piezoresistors is optimized for each diaphragm thickness in order to obtain the highest possible linearity of the output signal. The overall size of the sensing element die is $2 \mathrm{~mm} \times 2 \mathrm{~mm} \times 0.38 \mathrm{~mm}$. After fabrication, the die is anodically bonded to a glass support. A photograph of the sensing element mounted on a TO-5 housing is shown in Figure 1a. In order to make a pressure sensor suitable for industrial applications and to ensure optimal operating conditions for the sensing element, a special sensor body must be used. A sensing element packaged in such a body constitutes a pressure sensor. A photograph of an industrial-grade pressure sensor based on the SP 12 sensing element is given in Figure $1 \mathrm{~b}$.

Electrically, the SP 12 sensor is a Wheatstone bridge made of pressure-dependent resistors. It is technologically optimized for constant current excitation, $I_{0}$. That current generates the voltage $V_{\text {out }}$ at the sensor's output port, and $V_{b r}$ at its excitation port.
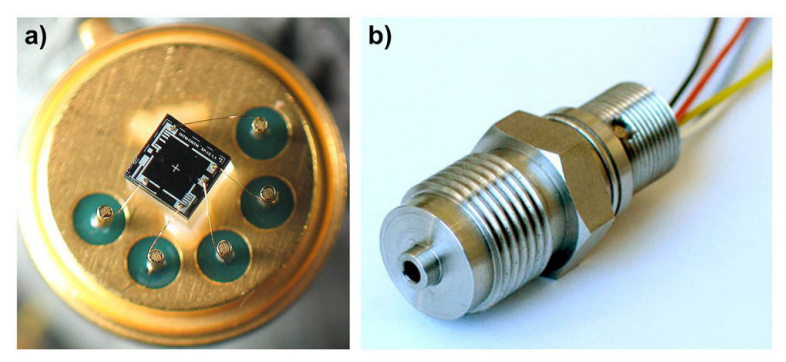

Figure 1. a) Photograph of the SP-12 sensing element mounted on a TO-5 housing, b) industrial pressure sensor based on the SP-12 sensing element

\section{Sensor Characterization}

In order to devise a method for simultaneous measurement of pressure and temperature by using a single piezoresistive pressure sensor, experimental charac- terization of a typical SP 12 sensor was performed. The main objective of the characterization was to determine the dependences of the two output parameters of the sensor $\left(R_{\text {out }}=V_{\text {out }} / I_{0}\right.$ and $\left.R_{b r}=V_{b r} / I_{0}\right)$ on both the applied pressure and the temperature.

A simplified block diagram of the measurement setup used for the sensor characterization is shown in Fig. 2. It consists of a high-performance automatic pressure calibrator (Mensor APC600), a temperature chamber (Vötsch VTL7010), a signal acquisition unit developed at ICTM-CMT specifically for this purpose (the dashed rectangle) and a personal computer. A software application is made for the computer to perform three main tasks: communication with both the signal acquisition unit and the pressure calibrator, user interface for measurement indication and instrument control, and data storage. The pressure sensor to be characterized is placed inside the temperature chamber. A high-performance resistive temperature sensor (Pt 100 , better than $\pm 0.06^{\circ} \mathrm{C}$ ) is attached to the surface of the pressure sensor, enabling the reference measurement of the pressure sensor's temperature.

The sensor characterization was performed at 10 different temperatures (from $70^{\circ} \mathrm{C}$ to $20^{\circ} \mathrm{C}$ ), starting from the highest temperature in order to avoid water condensation during the experiment. The nominal pressure range of the sensor under test was divided into 10 equal intervals, yielding 11 equidistant pressure values to be set.

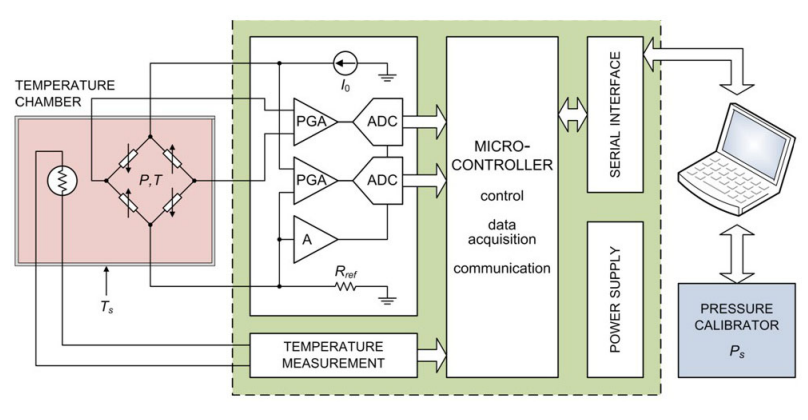

Figure 2. Block diagram of the experimental setup

Additionally, the pressure was set in two passes: first from the lowest to the highest value and then in the opposite direction, so that hysteresis effects could be observed. At each measurement point, the pressure indicated by the calibrator, $P$ [bar], the temperature chamber setpoint, $T_{s}\left[{ }^{\circ} \mathrm{C}\right]$, and the temperature of the sensor under test, $T\left[{ }^{\circ} \mathrm{C}\right]$, were recorded as well as the measured values of both sensor output parameters, $R_{\text {out }}$ and $R_{b r}$. The entire experiment produces 220 data points and lasts about 8 hours. 
In order to evaluate the performance of the tested sensor, an approach is chosen that reflects the most basic application of such sensors, which assumes only offset and span adjustment at the reference temperature. The indication of the measured pressure is given as

$$
\hat{P}\left(R_{\text {out }}, R_{\text {br }}\right)=a_{0}+a_{1} \cdot R_{\text {out }}\left(P, T_{\text {ref }}\right)
$$

where $a_{0}$ and $a_{1}$ are parameters calculated by fitting the linear function (1) to the experimental data acquired at the reference temperature, $T=T_{\text {ref }}=20^{\circ} \mathrm{C}$, using the method of least squares (Wolberg, 2006). The relative pressure measurement deviation from the pressure value indicated by the calibrator at the $i^{\text {th }}$ data point is expressed as a percentage of full scale, according to

$$
\delta P_{i}[\% \mathrm{FS}]=\frac{\hat{P}_{i}\left(R_{\text {out }}, R_{\text {bri }}\right)-P_{i}}{\left|P_{\max }-P_{\min }\right|} \cdot 100, i=1 \mathrm{~K} N
$$

where $\mathrm{N}$ is the total number of data points $(N=220)$, and $P_{\max }$ and $P_{\min }$ are the maximum and the minimum of the nominal pressure range, respectively $\left(P_{\max }=100 \mathrm{bar}\right.$, $P_{\min }=0$ bar $)$.

As a figure of merit of the tested sensor utilized for pressure measurement we use the expression $\overline{\delta P} \pm U_{P}$, where $\overline{\delta P}$ is the relative deviation mean value, and $U_{p}$ is the expanded uncertainty with a coverage factor of 2 (JCGM, 2008):

$$
\begin{gathered}
\overline{\delta P}=\frac{1}{N} \cdot \sum_{i=1}^{N} \delta P_{i}, \\
U_{P}=2 \cdot \sqrt{\frac{1}{N-1} \cdot \sum_{i=1}^{N}\left(\hat{P}_{i}-P_{i}\right)^{2}} \cdot \frac{100}{\left|P_{\max }-P_{\min }\right|} .
\end{gathered}
$$

This representation of results is a robust indicator that describes the deviation of measurement values from the values set by the calibrator as a standard.

A similar approach is used for temperature, obtaining the indication of the measured temperature as

$$
\hat{T}\left(R_{o u t}, R_{b r}\right)=b_{0}+b_{1} \cdot R_{b r}\left(P_{r e f}\right)
$$

where $b_{0}$ and $b_{1}$ are parameters calculated by fitting the linear function (5) to the experimental data acquired at the reference pressure, $P=P_{\text {ref }}=0$ bar (relative to the atmospheric pressure), using the method of least squares. The temperature measurement deviation at the $i^{\text {th }}$ data point is expressed in degrees Celsius, according to

$$
\Delta T_{i}\left[{ }^{\circ} \mathrm{C}\right]=\hat{T}_{i}\left(R_{\text {out } i}, R_{\text {bri }}\right)-T_{i}, i=1 \mathrm{~K} N .
$$

As a figure of merit of the tested sensor utilized for temperature measurement we use the expression $\overline{\Delta T} \pm U_{T}$, where $\overline{\Delta T}$ is the deviation mean value, and $U_{T}$ is the expanded uncertainty with the coverage factor of 2 (JCGM, 2008):

$$
\begin{gathered}
\overline{\Delta T}=\frac{1}{N} \cdot \sum_{i=1}^{N} \Delta T_{i}, \\
U_{T}=2 \cdot \sqrt{\frac{1}{N-1} \cdot \sum_{i=1}^{N}\left(\hat{T}_{i}-T_{i}\right)^{2}} .
\end{gathered}
$$

Here the current temperature measured by the Pt -100 sensor is taken as the standard and the measurement results are expressed as a deviation from that value. The numerical results showing the performance of the tested sensor, based on the characterization results are given in Table 1. These results confirm that an acceptable level of measurement performance cannot be achieved without an adequate sensor correction method. The method that will be presented here relies on the mathematical model of the sensor, and computer algorithm enabling real-time calculation of both the pressure and temperature value indication.

\section{Sensor Correction Method}

Based on the analysis of the experimentally obtained data, a parametric mathematical model of a silicon piezoresistive MEMS pressure sensor has been established, taking into account the influence of the pressure and the temperature on the sensor's output signals. In this case, the goal of mathematical modeling is to determine the sensor calibration functions, i.e. the dependence of the measurement indication of both the pressure and the temperature on the sensor's output parameters $R_{\text {out }}$ and $R_{b r}$. We have chosen a model consisting of two bivariate polynomials:

$$
\begin{aligned}
& \hat{P}\left(R_{\text {out }}, R_{b r}\right)=\sum_{j=0}^{m} \sum_{k=0}^{n} a_{j k} \cdot R_{\text {out }}^{j} \cdot R_{b r}^{k} \\
& \hat{T}\left(R_{\text {out }}, R_{b r}\right)=\sum_{j=0}^{u} \sum_{k=0}^{v} b_{j k} \cdot R_{\text {out }}^{j} \cdot R_{b r}^{k}
\end{aligned}
$$

where $\hat{P}$ and $\hat{T}$ are the pressure and the temperature indication, respectively, while $a_{j k}$ and $b_{j k}$ are the parameters to be determined for the given sensor. The values of $m, n, u$, and $v$, and consequentially the degrees of both polynomials, need to be chosen carefully in order to maximize the fidelity of the mathematical model, but keeping in mind the necessary calculation time in the target hardware. In this case, the following values have been chosen: $m=4, n=4, u=2$ and $v=4$. 
The parameters $a j k$ are calculated by fitting the function given by (10) to the characterization data $\left(P_{i}, R_{\text {out } i}, R_{b r i}\right.$, $i=1, \ldots, 220$ ), using the method of least squares (Wolberg, 2006). Similarly, the parameters $b_{j k}$ are calculated by fitting the function given by (11) to the characterization data $\left(T_{i}, R_{\text {out } i}, R_{b r i}, i=1, \ldots, 220\right)$. In this way, the mathematical model of each of the characterized sensors is determined, yielding the corresponding calibration functions for both pressure and temperature measurement. These functions enable simultaneous pressure and temperature measurements to be performed using a single piezoresistive MEMS pressure sensor.

\section{RESULTS AND DISCUSSION}

For the purpose of experimental verification of the proposed method, an intelligent industrial transmitter, developed at ICTM-CMT, was used as a hardware platform, with the tested sensor built-in. Its simplified block diagram is given in Figure 3. Conceptually, it replaces the measurement system used for sensor characterization (shown in Figure 2), but with two major differences: 1) the separate temperature sensor does not exist;2) the microcontroller block performs all the data processing (including sensor correction), control functions and communication via an industrial interface.

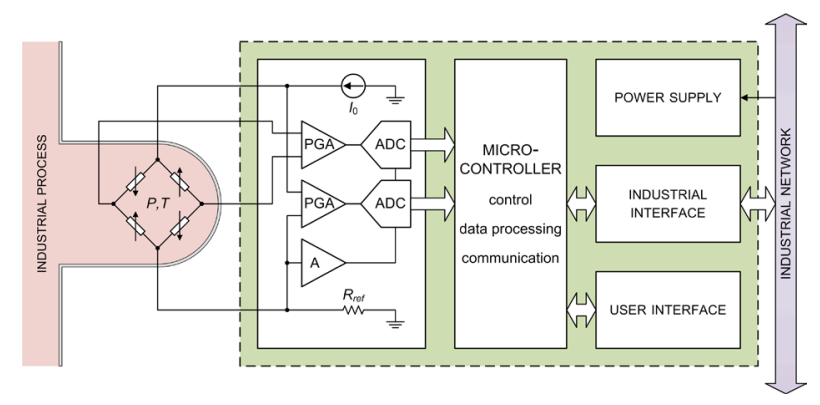

Figure 3. Simplified block diagram of an intelligent industrial instrument for simultaneous pressure and temperature measurement

The instrument performs real-time sensor corrections by calculating (10) and (11) for each set of data appearing at the outputs of the A/D converters and representing the values of $R_{\text {out }}$ and $R_{b r}$ simultaneously sampled at a certain rate. For that purpose, the parameters $a_{j k}$ and $b_{j k}$ of the given sensor must be stored in the instrument's non-volatile memory or, alternatively, in the sensor's own memory (IEEE 1451.4, 2004). The measurements were performed in a similar way as in the sensor characterization experiment, but this time the pressure and temperature measurement indications provided by the instrument were recorded at each measurement point. The relative pressure measurement deviation, expressed as a percentage of full scale according to (2), is shown in Fig. 4 for the tested sensor. The temperature measurement deviation, expressed in degrees Celsius according to (6), is shown in Figure 5.

Table 1 summarizes the pressure and temperature measurement performance achieved using the tested sensor without the sensor correction (based on characterization data), and with the described correction method applied (based on experimental verification data). When these values are compared, it becomes evident that the method enables a great improvement in both pressure and temperature measurement performance. The pressure measurement relative uncertainty is reduced by a factor of 65.3 , while the temperature uncertainty is reduced by a factor of 67.6 .

As the proposed measurement method is intended primarily for industrial applications, the main criterion for its applicability is the achievable measurement performance in comparison to that of existing industrial solutions. When pressure measurement is considered, modern industrial transmitters typically have a relative uncertainty of $0.075 \% \mathrm{FS}$ or worse, depending on the specified temperature range. The results shown in Table 1 indicate that such performance level can be exceeded by using the proposed measurement method, at least in the considered temperature range $\left(-20^{\circ} \mathrm{C}\right.$ to $\left.70^{\circ} \mathrm{C}\right)$.

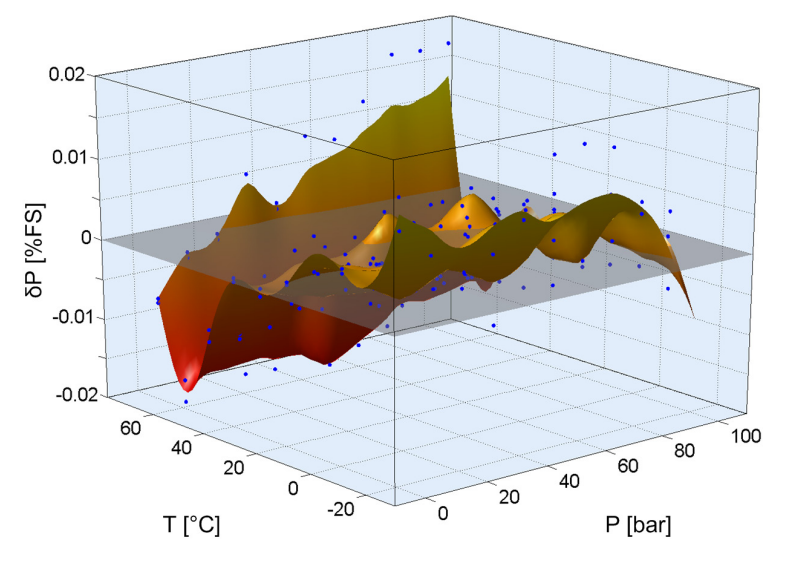

Figure 4. Relative pressure measurement deviation with the proposed method applied, as a function of pressure $P$ and temperature $T$ 


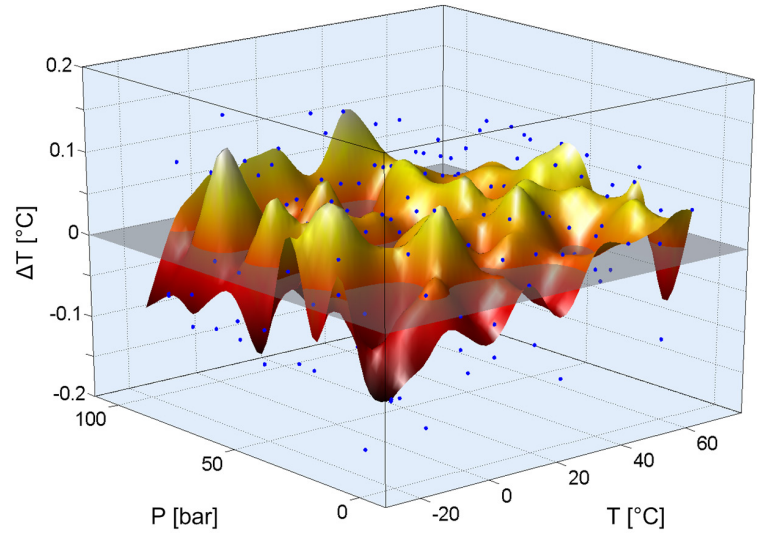

Figure 5. Temperature measurement deviation with the proposed method applied, as a function of temperature $T$ and the pressure $P$

Temperature measurements in industrial processes are commonly performed by using temperature probes based on thermocouples (TC) or resistance temperature detectors (RTD). The most commonly used TCs are the types J, $\mathrm{K}$, and $\mathrm{E}$, which can achieve $\pm 1.5^{\circ} \mathrm{C}$ in the temperature range from $-40^{\circ} \mathrm{C}$ to $375^{\circ} \mathrm{C}$ (Class 1 tolerance (IEC 60584-2, 1989)), but are usable up to $800^{\circ} \mathrm{C}$ (type J), $1300^{\circ} \mathrm{C}$ (type $\mathrm{K}$ ), and $900^{\circ} \mathrm{C}$ (type E). Also common are the type T TCs $\left( \pm 0.5^{\circ} \mathrm{C}\right.$ in the temperature range from $-40^{\circ} \mathrm{C}$ to $125^{\circ} \mathrm{C}$ ). The measurement uncertainty of industrial Class B platinum RTDs is given as $\left(0.3+0.005^{*}|T|\right)^{\circ} \mathrm{C}$, where $T$ is the measured temperature in the range from $-196^{\circ} \mathrm{C}$ to $600^{\circ} \mathrm{C}$ for wire-wound RTDs and $-50^{\circ} \mathrm{C}$ to $500^{\circ} \mathrm{C}$ for film RTDs (IEC 60751, 2008). In terms of temperature measurement performance, the method presented in this paper enabled achieving an expanded measurement uncertainty lower than $0.3^{\circ} \mathrm{C}$, which is much better than that of type J, Kand E TCs, better than that of type T TCs, and comparable to that of Class B RTDs. However, the temperature range of SP-12 sensing elements is limited by the physical properties of silicon to less than $125^{\circ} \mathrm{C}$. This limitation can be overcome by using pressure sensing elements based on SOI substrates (Kumar and Pant, 2014; Guo 2009).

\begin{tabular}{ccc}
\hline & $\begin{array}{c}\text { without sensor } \\
\text { correction }\end{array}$ & $\begin{array}{c}\text { with correction } \\
\text { applied }\end{array}$ \\
\hline $\bar{a} P \pm U_{P}[\% \mathrm{FS}]$ & $0.053 \pm 0.979$ & $-0.001 \pm 0.015$ \\
\hline$\overline{\Delta T} \pm U_{T}\left[{ }^{\circ} \mathrm{C}\right]$ & $3.222 \pm 8.520$ & $0.010 \pm 0.126$ \\
\hline
\end{tabular}

Table 1. Pressure \& Temperature Measurement Performance $\left(P_{\min }=0\right.$ bar, $P_{\max }=100 \mathrm{bar}, T_{\min }=-20^{\circ} \mathrm{C}$, $T_{\max }=70^{\circ} \mathrm{C}, m=4, n=4, u=2$ and $\left.v=4\right)$
Based on the described intelligent instrument architecture (see Figure 3), and the presented measurement method, two products are developed and produced at the Center of Microelectronic Technologies. The first is the intelligent industrial pressure transmitter, shown in Figure 6a, and the second is the intelligent industrial liquid level transmitter, shown in Figure 6b. They share the same modular hardware platform, which enables the use of up to 2 piezoresistive MEMS pressure sensors, both capable of measuring pressure and/or temperature. The connection with an industrial control system is established via a 2-wire cable. It enables the use of the standard analog $4 \mathrm{~mA}$ to $20 \mathrm{~mA}$ signal (represents the value of the measured quantity and provides the power to the transmitter), as well as a 2-way digital communication (based on a superimposed modulated signal and a standardized industrial protocol). The performance and reliability of both products have been proven in harsh industrial environments.

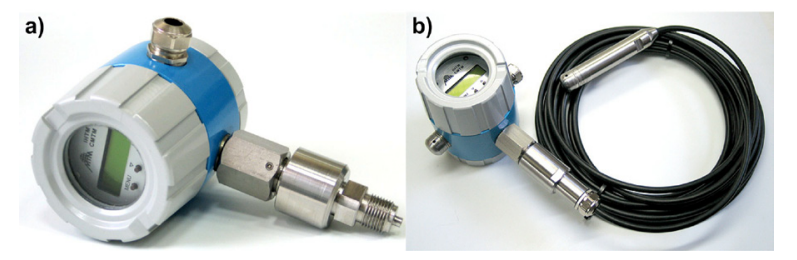

Figure 6. a) Intelligent pressure transmitter TPrs-111 and b) intelligent liquid level transmitter TPns-101

\section{CONCLUSION}

In this paper an overview is given of MEMS pressure sensors and the current state of the art in the field of industrial instrumentation. A novel measurement method is described that enables simultaneous pressure and temperature measurements to be performed using a single silicon piezoresistive MEMS pressure sensor. The presented results show that the achieved pressure measurement performance $\left(<0.03 \% \mathrm{FS},-20^{\circ} \mathrm{C}<T<70^{\circ} \mathrm{C}\right)$ is more than adequate for use in high performance industrial pressure transmitters. At the same time, the temperature measurement performance $\left(<0.3^{\circ} \mathrm{C}, 0 \mathrm{bar}<P<100 \mathrm{bar}\right)$ is better than that achievable with thermocouples, and comparable to Class B platinum resistive thermometers. Although the temperature range is limited by the physical properties of silicon (up to $125^{\circ} \mathrm{C}$ ), it is adequate for numerous applications. This limitation can be overcome by using sensing elements based on SOI substrates. Apart from simultaneous pressure and temperature measurement at 
the same point in an industrial process, some other useful applications can be envisioned, including validation of a separate temperature sensor, temperature compensation or sensor diagnostics in multisensor systems etc. As the proposed measurement method relies on the existing concept of intelligent industrial pressure transmitters with piezoresistive sensors, some existing types of such transmitters can be upgraded so to include temperature measurement capability at a minimal additional cost.

\section{REFERENCES}

Balavalad, K.B., \& Sheeparamatti, B.G. (2015). A Critical Review of MEMS Capacitive Pressure Sensors. Sensors \& Transducers 187(4), 120-128.

Djurić Z., Matović, J., Matić, M., Mišović (Simičić), N., Petrović, R., Smiljanić, M., \& Lazić, Ž. (1986). Pressure Sensor with Silicon Diaphragm. In XIV Yugoslav Conference on Microelectronics MIEL, 1986 (pp. 88-100). Belgrade, Yugoslavia.

Djurić, Z. (1995). Rezultati istraživanja i razvoja Si senzora i transmitera pritiska u IHTM - Centru za mikroelektronske tehnologije i monokristale. In $20^{\text {th }}$ International Conference on Microelectronics MIEL, 1995 Niš, Yugoslavia. (in Serbian).

Doelle, M.B. (2011). Temperature Extraction from a Pressure Sensor. US Patent No. 8,082,796 B1 Silicon Microstructures Inc., USA.

Frantlović, M., Jokić, I., \& Nešić, D. (2007). A Wireless System for Liquid Level Measurement. In $8^{\text {th }}$ International Conference on Telecommunication in Modern Satellite, Cable and Broadcasting Services TELSIKS, 26-28 September 2007 (pp. 475-478). Niš, Serbia: IEEE.

Frantlović, M., Jovanov, V., \& Miljković, B. (2009). Intelligent Industrial Transmitters of Pressure and Other Process Parameters. Telfor Journal 1(2), 65-8.

Frantlović, M., Jokić, I., Djurić, Z., \& Radulović, K. (2013). Analysis of the competitive adsorption and mass transfer influence on equilibrium mass fluctuations in affinity-based biosensors. Sensors and Actuators B: Chemical 189, 71-79. DOI: 10.1016/j. snb.2012.12.080.

Frantlović M., Jokić, I., Lazić, Ž., Vukelić, B., Obradov, M., \& Vasiljević-Radović, D. Temperature measurement using silicon piezoresistive MEMS pressure sensors. In $29^{\text {th }}$ International Conference on Microelectronics MIEL, 12-15 May 2014 (pp. 159-161). Belgrade, Serbia: IEEE.

Guo, T., Qiao, X.G., Jia, Z.N., Zhao, Q.D., \& Dong, X.Y. (2006). Simultaneous measurement of temperature and pressure by a single fiber Bragg grating with a broadened reflection spectrum. Appl. Opt. 45, 2935-2939. DOI: 10.1364/AO.45.002935.
Guo, S., Eriksen, H., Childress, K., Fink, A., \& Hoffman, M. (2009). High temperature smart-cut SOI pressure sensor. Sens. Actuators A 154, 255-260. DOI: 10.1016/j.sna.2009.03.01.

IEC 607512008 Industrial platinum resistance thermometers and platinum temperature sensors (International Electrotechnical Commission).

IEC 60584-2 1989 Thermocouples - Part 2: Tolerances (International Electrotechnical Commission).

IEEE 1451.4 2004 IEEE Standard for a Smart Transducer Interface for Sensors and Actuators: Mixed-Mode Communication Protocols and Transducer Electronic Data Sheet (TEDS) Formats (Institute of Electrical and Electronics Engineers).

JCGM 2008 Evaluation of measurement data - Guide to the expression of uncertainty in measurement (GUM) JCGM 100:2008 Joint Committee for Guides in Metrology.

Kumar, S.S., \& Pant, B.D. (2014). Design principles and considerations for the 'ideal' silicon piezoresistive pressure sensor: a focused review. Microsystem Technologies 20(7), 1213-1247. DOI: 10.1007/ s00542-014-2215-7.

Matić, M., Lazić, Ž., Radulović, K., Smiljanić, M.M., \& Rašljić, M. (2013). Eksperimentalno određivanje optimalne linearnosti senzora pritiska. In $57^{\text {th }}$ Conference for Electronics, Telecommunications, Computers, Automation and Nuclear Engineering ETRAN, 3-6 Jun 2013 (pp. MO3.1). Zlatibor, Serbia.

Matović J., Djurić, Z., Simičić, N., Matić, M., \& Petrović, R. (1991). A Nonlinear Simulation of Pressure Sensor. In $19^{\text {th }}$ Yugoslav Conference on Microelectronics MIEL, 1991. Belgrade, Yugoslavia.

Rivera, J., Carrillo, M., Chacón, M., Herrera, G., \& Bojorquez, G. (2007). Self-Calibration and Optimal Response in Intelligent Sensors Design Based on Artificial Neural Networks. Sensors 7, pp. 15091529.

Smiljanić, M.M., Lazić, Ž., Djurić, Z., \& Radulović, K. (2007). Dizajn i modelovanje modifikovanog senzora niskih pritisaka SP-6 IHTM-CMTM. In 51 $1^{\text {st }}$ Conference for Electronics, Telecommunications, Computers, Automation and Nuclear Engineering ETRAN, 4-8 June 2007 (pp. MO3.2-1-4).Herceg Novi, Montenegro. (in Serbian).

Smiljanić M.M., Jović, V., \& Lazić, Ž. (2012). Maskless convex corner compensation technique on a (1 $00)$ silicon substrate in a $25 \mathrm{wt} \% \mathrm{TMAH}$ water solution. Journal of Micromechanics and Microengineering 22(11), 115011. DOI: 10.1088/09601317/22/11/115011.

Wolberg, J. (2006). Data Analysis Using the Method of Least Squares. Berlin, Heidelberg: Springer-Verlag. 